\title{
Operadic representations of harmonic oscillator in some 3d algebras
}

\author{
Eugen PAAL and Jüri VIRKEPU \\ Department of Mathematics, Tallinn University of Technology \\ Ehitajate tee 5, 19086 Tallinn, Estonia \\ E-mails: eugen.paal@ttu.ee and jvirkepu@staff.ttu.ee
}

\begin{abstract}
It is explained how the time evolution of the operadic variables may be introduced by using the operadic Lax equation. The operadic Lax representations for the harmonic oscillator are constructed in 3-dimensional binary anti-commutative algebras. As an example, an operadic Lax representation for the harmonic oscillator in the Lie algebra $\mathfrak{s l}(2)$ is constructed.
\end{abstract}

2000 MSC: 18D50, 70G60

\section{Introduction}

In Hamiltonian formalism, a mechanical system is described by the canonical variables $q^{i}, p_{i}$ and their time evolution is prescribed by the Hamiltonian system

$$
\frac{d q^{i}}{d t}=\frac{\partial H}{\partial p_{i}}, \quad \frac{d p_{i}}{d t}=-\frac{\partial H}{\partial q^{i}}
$$

By a Lax representation $[3,1]$ of a mechanical system one means such a pair $(L, M)$ of matrices (linear operators) $L, M$ that the above Hamiltonian system may be represented as the Lax equation

$$
\frac{d L}{d t}=M L-L M
$$

Thus, from the algebraic point of view, mechanical systems can be described by linear operators, i.e by linear maps $V \rightarrow V$ of a vector space $V$. As a generalization of this one can pose the following question [4]: how to describe the time evolution of the linear operations (multiplications) $V^{\otimes n} \rightarrow V ?$

The algebraic operations (multiplications) can be seen as an example of the operadic variables [2]. If an operadic system depends on time one can speak about operadic dynamics [4]. The latter may be introduced by simple and natural analogy with the Hamiltonian dynamics. In particular, the time evolution of the operadic variables may be given by the operadic Lax equation. In $[5,6]$, a 2-dimensional binary operadic Lax representation for the harmonic oscillator was constructed. In the present paper we construct the operadic Lax representations for the harmonic oscillator in 3-dimensional binary anti-commutative algebras. As an example, an operadic Lax representation for the harmonic oscillator in the Lie algebra $\mathfrak{s l}(2)$ is constructed. 


\section{Endomorphism operad and Gerstenhaber brackets}

Let $K$ be a unital associative commutative ring, $V$ be a unital $K$-module, and $\mathcal{E}_{V}^{n}:=\mathcal{E} n d_{V}^{n}:=$ $\operatorname{Hom}\left(V^{\otimes n}, V\right)(n \in \mathbb{N})$. For an operation $f \in \mathcal{E}_{V}^{n}$, we refer to $n$ as the degree of $f$ and often write (when it does not cause confusion) $f$ instead of $\operatorname{deg} f$. For example, $(-1)^{f}:=(-1)^{n}, \mathcal{E}_{V}^{f}:=\mathcal{E}_{V}^{n}$ and $\circ_{f}:=\circ_{n}$. Also, it is convenient to use the reduced degree $|f|:=n-1$. Throughout this paper, we assume that $\otimes:=\otimes_{K}$.

Definition 2.1 (endomorphism operad [2]). For $f \otimes g \in \mathcal{E}_{V}^{f} \otimes \mathcal{E}_{V}^{g}$ define the partial compositions

$$
f \circ \circ_{i} g:=(-1)^{i|g|} f \circ\left(\mathrm{id}_{V}^{\otimes i} \otimes g \otimes \mathrm{id}_{V}^{\otimes(|f|-i)}\right) \quad \in \mathcal{E}_{V}^{f+|g|}, \quad 0 \leq i \leq|f|
$$

The sequence $\mathcal{E}_{V}:=\left\{\mathcal{E}_{V}^{n}\right\}_{n \in \mathbb{N}}$, equipped with the partial compositions $\circ_{i}$, is called the endomorphism operad of $V$.

Definition 2.2 (total composition [2]). The total composition $\bullet: \mathcal{E}_{V}^{f} \otimes \mathcal{E}_{V}^{g} \rightarrow \mathcal{E}_{V}^{f+|g|}$ is defined by

$$
f \bullet g:=\sum_{i=0}^{|f|} f \circ_{i} g \quad \in \mathcal{E}_{V}^{f+|g|}, \quad|\bullet|=0
$$

The pair $\operatorname{Com} \mathcal{E}_{V}:=\left\{\mathcal{E}_{V}, \bullet\right\}$ is called the composition algebra of $\mathcal{E}$.

Definition 2.3 (Gerstenhaber brackets [2]). The Gerstenhaber brackets $[\cdot, \cdot]$ are defined in $\operatorname{Com} \mathcal{E}_{V}$ as a graded commutator by

$$
[f, g]:=f \bullet g-(-1)^{|f||g|} g \bullet f=-(-1)^{|f||g|}[g, f], \quad|[\cdot, \cdot]|=0
$$

The commutator algebra of $\operatorname{Com} \mathcal{E}_{V}$ is denoted as $\operatorname{Com}^{-} \mathcal{E}_{V}:=\left\{\mathcal{E}_{V},[\cdot, \cdot]\right\}$. One can prove (e.g [2]) that $\mathrm{Com}^{-} \mathcal{E}_{V}$ is a graded Lie algebra. The Jacobi identity reads

$$
(-1)^{|f||h|}[[f, g], h]+(-1)^{|g||f|}[[g, h], f]+(-1)^{|h||g|}[[h, f], g]=0
$$

\section{Operadic Lax equation and harmonic oscillator}

Assume that $K:=\mathbb{R}$ or $K:=\mathbb{C}$ and operations are differentiable. Dynamics in operadic systems (operadic dynamics) may be introduced by

Definition 3.1 (operadic Lax pair [4]). Allow a classical dynamical system to be described by the Hamiltonian system (1.1). An operadic Lax pair is a pair $(L, M)$ of linear operations $L, M \in \mathcal{E}_{V}$, such that the Hamiltonian system (1.1) may be represented as the operadic Lax equation

$$
\frac{d L}{d t}=[M, L]:=M \bullet L-(-1)^{|M||L|} L \bullet M
$$

Evidently, the degree constraints $|M|=|L|=0$ give rise to ordinary Lax equation $(1.2)[3,1]$. 
The Hamiltonian of the harmonic oscillator is

$$
H(q, p)=\frac{1}{2}\left(p^{2}+\omega^{2} q^{2}\right)
$$

Thus, the Hamiltonian system of the harmonic oscillator reads

$$
\frac{d q}{d t}=\frac{\partial H}{\partial p}=p, \quad \frac{d p}{d t}=-\frac{\partial H}{\partial q}=-\omega^{2} q
$$

If $\mu$ is a linear algebraic operation we can use the above Hamilton equations to obtain

$$
\frac{d \mu}{d t}=\frac{\partial \mu}{\partial q} \frac{d q}{d t}+\frac{\partial \mu}{\partial p} \frac{d p}{d t}=p \frac{\partial \mu}{\partial q}-\omega^{2} q \frac{\partial \mu}{\partial p}=[M, \mu]
$$

Therefore, we get the following linear partial differential equation for $\mu(q, p)$ :

$$
p \frac{\partial \mu}{\partial q}-\omega^{2} q \frac{\partial \mu}{\partial p}=[M, \mu]
$$

By integrating (3.2) one can get sequences of operations called the operadic (Lax representations for) harmonic oscillator. Since the general solution of the partial differential equations depends on arbitrary functions, these representations are not uniquely determined.

\section{Evolution of binary algebras}

Let $A:=\{V, \mu\}$ be a binary algebra with an operation $x y:=\mu(x \otimes y)$. For simplicity assume that $|M|=0$. We require that $\mu=\mu(q, p)$ so that $(\mu, M)$ is an operadic Lax pair, i.e the Hamiltonian system (3.1) of the harmonic oscillator may be written as the operadic Lax equation

$$
\dot{\mu}=[M, \mu]:=M \bullet \mu-\mu \bullet M, \quad|\mu|=1, \quad|M|=0
$$

Let $x, y \in V$. Assuming that $|M|=0$ and $|\mu|=1$ we have

$$
\begin{aligned}
& M \bullet \mu=\sum_{i=0}^{0} M \circ_{i} \mu=M \circ_{0} \mu=M \circ \mu \\
& \mu \bullet M=\sum_{i=0}^{1} \mu \circ_{i} M=\mu \circ_{0} M+\mu \circ_{1} M=\mu \circ\left(M \otimes \operatorname{id}_{V}\right)+\mu \circ\left(\operatorname{id}_{V} \otimes M\right)
\end{aligned}
$$

Therefore,

$$
\frac{d}{d t}(x y)=M(x y)-(M x) y-x(M y)
$$

Let $\operatorname{dim} V=n$. In a basis $\left\{e_{1}, \ldots, e_{n}\right\}$ of $V$, the structure constants $\mu_{j k}^{i}$ of $A$ are defined by

$$
\mu\left(e_{j} \otimes e_{k}\right):=\mu_{j k}^{i} e_{i}, \quad j, k=1, \ldots, n
$$

In particular,

$$
\frac{d}{d t}\left(e_{j} e_{k}\right)=M\left(e_{j} e_{k}\right)-\left(M e_{j}\right) e_{k}-e_{j}\left(M e_{k}\right)
$$

By denoting $M e_{i}:=M_{i}^{s} e_{s}$, it follows that

$$
\dot{\mu}_{j k}^{i}=\mu_{j k}^{s} M_{s}^{i}-M_{j}^{s} \mu_{s k}^{i}-M_{k}^{s} \mu_{j s}^{i}, \quad i, j, k=1, \ldots, n
$$


E. Paal and J. Virkepu

\section{Main Theorem}

\section{Lemma 5.1. Matrices}

$$
L:=\left(\begin{array}{ccc}
p & \omega q & 0 \\
\omega q & -p & 0 \\
0 & 0 & 1
\end{array}\right), \quad M:=\frac{\omega}{2}\left(\begin{array}{ccc}
0 & -1 & 0 \\
1 & 0 & 0 \\
0 & 0 & 0
\end{array}\right)
$$

give a 3-dimensional Lax representation for the harmonic oscillator.

Lemma 5.2. Let $\operatorname{dim} V=3$ and $M$ be defined as in Lemma 5.1. Then the 3-dimensional binary operadic Lax equations read

$$
\left\{\begin{array}{lll}
\dot{\mu}_{11}^{1}=-\frac{\omega}{2}\left(\mu_{11}^{2}+\mu_{12}^{1}+\mu_{21}^{1}\right), & \dot{\mu}_{13}^{1}=-\frac{\omega}{2}\left(\mu_{13}^{2}+\mu_{23}^{1}\right), & \dot{\mu}_{33}^{1}=-\frac{\omega}{2} \mu_{33}^{2} \\
\dot{\mu}_{12}^{1}=-\frac{\omega}{2}\left(\mu_{12}^{2}-\mu_{11}^{1}+\mu_{22}^{1}\right), & \dot{\mu}_{23}^{1}=-\frac{\omega}{2}\left(\mu_{23}^{2}-\mu_{13}^{1}\right), & \dot{\mu}_{33}^{2}=\frac{\omega}{2} \mu_{33}^{1} \\
\dot{\mu}_{21}^{1}=-\frac{\omega}{2}\left(\mu_{21}^{2}-\mu_{11}^{1}+\mu_{22}^{1}\right), & \dot{\mu}_{31}^{1}=-\frac{\omega}{2}\left(\mu_{31}^{2}+\mu_{32}^{1}\right), & \dot{\mu}_{13}^{3}=-\frac{\omega}{2} \mu_{23}^{3} \\
\dot{\mu}_{22}^{1}=-\frac{\omega}{2}\left(\mu_{22}^{2}-\mu_{12}^{1}-\mu_{21}^{1}\right), & \dot{\mu}_{32}^{1}=-\frac{\omega}{2}\left(\mu_{32}^{2}-\mu_{31}^{1}\right), & \dot{\mu}_{23}^{3}=\frac{\omega}{2} \mu_{13}^{3} \\
\dot{\mu}_{11}^{2}=\frac{\omega}{2}\left(\mu_{11}^{1}-\mu_{12}^{2}-\mu_{21}^{2}\right), & \dot{\mu}_{13}^{2}=-\frac{\omega}{2}\left(\mu_{23}^{2}-\mu_{13}^{1}\right), & \dot{\mu}_{22}^{3}=\frac{\omega}{2}\left(\mu_{12}^{3}+\mu_{21}^{3}\right) \\
\dot{\mu}_{12}^{2}=\frac{\omega}{2}\left(\mu_{12}^{1}+\mu_{11}^{2}-\mu_{22}^{2}\right), & \dot{\mu}_{23}^{2}=\frac{\omega}{2}\left(\mu_{23}^{1}+\mu_{13}^{2}\right), & \dot{\mu}_{21}^{3}=\frac{\omega}{2}\left(\mu_{11}^{3}-\mu_{22}^{3}\right) \\
\dot{\mu}_{21}^{2}=\frac{\omega}{2}\left(\mu_{21}^{1}+\mu_{11}^{2}-\mu_{22}^{2}\right), & \dot{\mu}_{31}^{2}=-\frac{\omega}{2}\left(\mu_{32}^{2}-\mu_{31}^{1}\right), & \dot{\mu}_{11}^{3}=-\frac{\omega}{2}\left(\mu_{21}^{3}+\mu_{12}^{3}\right) \\
\dot{\mu}_{22}^{2}=\frac{\omega}{2}\left(\mu_{22}^{1}+\mu_{12}^{2}+\mu_{21}^{2}\right), & \dot{\mu}_{32}^{2}=\frac{\omega}{2}\left(\mu_{32}^{1}+\mu_{31}^{2}\right), & \dot{\mu}_{12}^{3}=\frac{\omega}{2}\left(\mu_{11}^{3}-\mu_{22}^{3}\right) \\
\dot{\mu}_{33}^{3}=0, & \dot{\mu}_{32}^{3}=\frac{\omega}{2} \mu_{31}^{3}, & \dot{\mu}_{31}^{3}=-\frac{\omega}{2} \mu_{32}^{3}
\end{array}\right.
$$

In what follows, consider only anti-commutative algebras. Then one has

Corollary 5.3. Let $A$ be a 3-dimensional anti-commutative algebra, i.e

$$
\mu_{j k}^{i}=-\mu_{k j}^{i}, \quad i, j, k=1,2,3
$$

Then the operadic Lax equations for the harmonic oscillator read

$$
\left\{\begin{array}{lll}
\dot{\mu}_{12}^{1}=-\frac{\omega}{2} \mu_{12}^{2}, & \dot{\mu}_{12}^{2}=\frac{\omega}{2} \mu_{12}^{1}, & \dot{\mu}_{12}^{3}=0 \\
\dot{\mu}_{13}^{1}=-\frac{\omega}{2}\left(\mu_{23}^{1}+\mu_{13}^{2}\right), & \dot{\mu}_{13}^{2}=-\frac{\omega}{2}\left(\mu_{23}^{2}-\mu_{13}^{1}\right), & \dot{\mu}_{13}^{3}=-\frac{\omega}{2} \mu_{23}^{3} \\
\dot{\mu}_{23}^{1}=\frac{\omega}{2}\left(\mu_{13}^{1}-\mu_{23}^{2}\right), & \dot{\mu}_{23}^{2}=\frac{\omega}{2}\left(\mu_{13}^{2}+\mu_{23}^{1}\right), & \dot{\mu}_{23}^{3}=\frac{\omega}{2} \mu_{13}^{3}
\end{array}\right.
$$

For the harmonic oscillator, define its auxiliary functions $A_{ \pm}$by

$$
A_{+}^{2}+A_{-}^{2}=2 \sqrt{2 H}, \quad A_{+}^{2}-A_{-}^{2}=2 p, \quad A_{+} A_{-}=\omega q
$$

Theorem 5.4. Let $C_{\nu} \in \mathbb{R}(\nu=1, \ldots, 9)$ be arbitrary real-valued parameters, such that

$$
C_{2}^{2}+C_{3}^{2}+C_{5}^{2}+C_{6}^{2}+C_{7}^{2}+C_{8}^{2} \neq 0
$$


Let $M$ be defined as in Lemma 5.1, and

$$
\left\{\begin{array}{l}
\mu_{11}^{1}=\mu_{22}^{1}=\mu_{33}^{1}=\mu_{11}^{2}=\mu_{22}^{2}=\mu_{33}^{2}=\mu_{11}^{3}=\mu_{22}^{3}=\mu_{33}^{3}=0 \\
\mu_{23}^{1}=-\mu_{32}^{1}=C_{2} p-C_{3} \omega q-C_{4} \\
\mu_{13}^{2}=-\mu_{31}^{2}=C_{2} p-C_{3} \omega q+C_{4} \\
\mu_{31}^{1}=-\mu_{13}^{1}=C_{2} \omega q+C_{3} p-C_{1} \\
\mu_{23}^{2}=-\mu_{32}^{2}=C_{2} \omega q+C_{3} p+C_{1} \\
\mu_{12}^{1}=-\mu_{21}^{1}=C_{5} A_{+}+C_{6} A_{-} \\
\mu_{12}^{2}=-\mu_{21}^{2}=C_{5} A_{-}-C_{6} A_{+} \\
\mu_{13}^{3}=-\mu_{31}^{3}=C_{7} A_{+}+C_{8} A_{-} \\
\mu_{23}^{3}=-\mu_{32}^{3}=C_{7} A_{-}-C_{8} A_{+} \\
\mu_{12}^{3}=-\mu_{21}^{3}=C_{9}
\end{array}\right.
$$

Then $(\mu, M)$ is a 3-dimensional anti-commutative binary operadic Lax pair for the harmonic oscillator.

Proof. Denote

$$
\begin{cases}G_{+}^{\omega}:=\dot{p}+\omega^{2} q, & G_{+}^{\omega / 2}:=\dot{A}_{+}+\frac{\omega}{2} A_{-} \\ G_{-}^{\omega}:=\omega(\dot{q}-p), & G_{-}^{\omega / 2}:=\dot{A}_{-}-\frac{\omega}{2} A_{+}\end{cases}
$$

Define the matrix

$$
\Gamma=\left(\Gamma_{\alpha}^{\beta}\right):=\left(\begin{array}{ccccccccc}
0 & 0 & 0 & 0 & 0 & 0 & 0 & 0 & 0 \\
G_{+}^{\omega} & G_{+}^{\omega} & G_{-}^{\omega} & G_{-}^{\omega} & 0 & 0 & 0 & 0 & 0 \\
-G_{-}^{\omega} & -G_{-}^{\omega} & G_{+}^{\omega} & G_{+}^{\omega} & 0 & 0 & 0 & 0 & 0 \\
0 & 0 & 0 & 0 & 0 & 0 & 0 & 0 & 0 \\
0 & 0 & 0 & 0 & G_{+}^{\omega / 2} & G_{-}^{\omega / 2} & 0 & 0 & 0 \\
0 & 0 & 0 & 0 & G_{-}^{\omega / 2} & -G_{+}^{\omega / 2} & 0 & 0 & 0 \\
0 & 0 & 0 & 0 & 0 & 0 & G_{+}^{\omega / 2} & G_{-}^{\omega / 2} & 0 \\
0 & 0 & 0 & 0 & 0 & 0 & G_{-}^{\omega / 2} & -G_{+}^{\omega / 2} & 0 \\
0 & 0 & 0 & 0 & 0 & 0 & 0 & 0 & 0
\end{array}\right)
$$

Then it follows from Corollary 5.3 that the 3 -dimensional anti-commutative binary operadic Lax equations read

$$
C_{\beta} \Gamma_{\alpha}^{\beta}=C_{2} \Gamma_{\alpha}^{2}+C_{3} \Gamma_{\alpha}^{3}+C_{5} \Gamma_{\alpha}^{5}+C_{6} \Gamma_{\alpha}^{6}+C_{7} \Gamma_{\alpha}^{7}+C_{8} \Gamma_{\alpha}^{8}=0, \quad \alpha=1, \ldots, 9
$$

Since the parameters $C_{\beta}(\beta=2,3,5,6,7,8)$ are arbitrary, not simultaneously zero, the latter constraints imply $\Gamma=0$.

Thus we have to consider the following differential equations

$$
G_{ \pm}^{\omega}=0=G_{ \pm}^{\omega / 2}
$$

We show that

$$
G_{ \pm}^{\omega}=0 \quad \Longleftrightarrow \quad\left\{\begin{array}{l}
\dot{p}=-\omega^{2} q \quad \Longleftrightarrow \quad(I I) \\
\dot{q}=p
\end{array} \quad G_{ \pm}^{\omega / 2}=0\right.
$$

First note that $(I)$ immediately follows from the definition of $G_{ \pm}^{\omega}$.

The proof of $(I I)$ can be found in [6] (Theorem $5.2(I)$ ). 
E. Paal and J. Virkepu

\section{Initial conditions and dynamical deformations}

It seems attractive to specify the coefficients $C_{\nu}$ in Theorem 5.4 by the initial conditions

$$
\left.\mu\right|_{t=0}=\stackrel{\circ}{\mu},\left.\quad p\right|_{t=0}=p_{0} \neq 0,\left.\quad q\right|_{t=0}=0
$$

The latter together with (5.1) yield the initial conditions for $A_{ \pm}$:

$$
\left\{\begin{array} { l } 
{ ( A _ { + } ^ { 2 } + A _ { - } ^ { 2 } ) | _ { t = 0 } = 2 | p _ { 0 } | } \\
{ ( A _ { + } ^ { 2 } - A _ { - } ^ { 2 } ) | _ { t = 0 } = 2 p _ { 0 } } \\
{ A _ { + } A _ { - } | _ { t = 0 } = 0 }
\end{array} \Longleftrightarrow \left\{\begin{array} { l } 
{ p _ { 0 } > 0 } \\
{ A _ { + } | _ { t = 0 } = \pm \sqrt { 2 p _ { 0 } } } \\
{ A _ { - } | _ { t = 0 } = 0 }
\end{array} \vee \vee \left\{\begin{array}{l}
p_{0}<0 \\
\left.A_{+}\right|_{t=0}=0 \\
\left.A_{-}\right|_{t=0}= \pm \sqrt{-2 p_{0}}
\end{array}\right.\right.\right.
$$

In what follows assume that $p_{0}>0$ and $\left.A_{+}\right|_{t=0}>0$. Other cases can be treated similarly.

From (5.3) we get the following linear system:

$$
\left\{\begin{array}{lll}
\stackrel{\circ}{\mu}_{23}^{1}=C_{2} p_{0}-C_{4}, & \stackrel{\circ}{\mu_{31}^{1}}=C_{3} p_{0}-C_{1}, & \stackrel{\circ}{\mu_{12}^{1}}=C_{5} \sqrt{2 p_{0}} \\
\stackrel{\circ}{\mu}_{13}^{2}=C_{2} p_{0}+C_{4}, & \stackrel{\circ}{\mu_{12}^{2}}=-C_{6} \sqrt{2 p_{0}}, & \stackrel{\circ}{\mu_{23}^{2}}=C_{3} p_{0}+C_{1} \\
\stackrel{\circ}{\mu}_{13}^{3}=C_{7} \sqrt{2 p_{0}}, & \stackrel{\circ}{\mu_{23}^{3}}=-C_{8} \sqrt{2 p_{0}}, & \stackrel{\circ}{\mu_{12}^{3}}=C_{9}
\end{array}\right.
$$

One can easily check that the latter system can be uniquely solved with respect to $C_{\nu}(\nu=$ $1, \ldots, 9)$ :

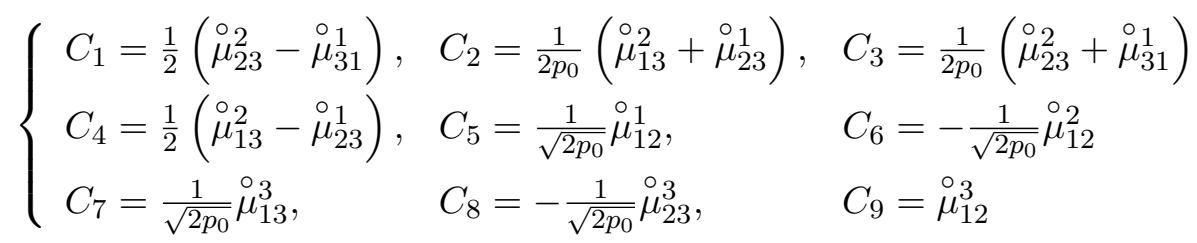

Remark 6.1. Note that the parameters $C_{\nu}$ have to satisfy condition (5.2) to get the operadic Lax representation for the harmonic oscillaror.

Definition 6.2. If $\mu \neq \stackrel{\circ}{\mu}$, then the multiplication $\mu$ is called a dynamical deformation of $\stackrel{\circ}{\mu}$ (over the harmonic oscillator). If $\mu=\stackrel{\circ}{\mu}$, then the multiplication $\stackrel{\circ}{\mu}$ is called dynamically rigid (over the harmonic oscillator).

\section{$7 \quad$ Examples}

Example 7.1 $(\mathfrak{s o}(3))$. As an example consider the Lie algebra $\mathfrak{s o}(3)$ with the structure equations

$$
\left[e_{1}, e_{2}\right]=e_{3}, \quad\left[e_{2}, e_{3}\right]=e_{1}, \quad\left[e_{3}, e_{1}\right]=e_{2}
$$

Thus, the nonzero structure constants are

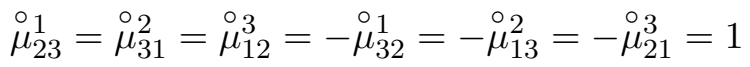

Using the above initial conditions (6.1), we get

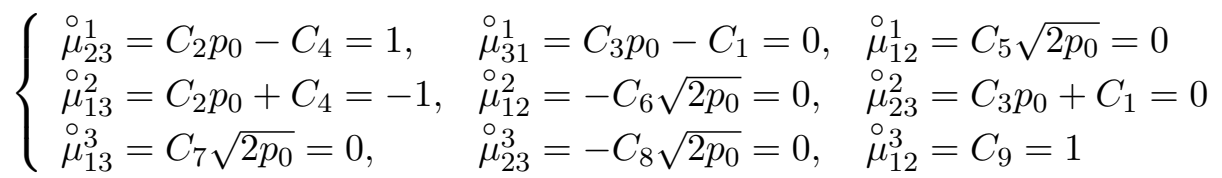


From this linear system it is easy to see that the only nontrivial constants are $C_{9}=-C_{4}=1$. Replacing these constants into (5.3) we get

$$
\mu_{j k}^{i}=\stackrel{\circ}{\mu}_{j k}^{i}, \quad i, j, k=1,2,\left.3 \quad \Longrightarrow \quad \dot{\mu}\right|_{\mathfrak{s o}(3)}=0
$$

Thus we can see that the present selection of the parameters $C_{\nu}(\nu=1, \ldots 9)$ via the structure constants of $\mathfrak{s o}(3)$ does not give rise to the operadic Lax representation for the harmonic oscillator. Thus $\mathfrak{s o}(3)$ is dynamically rigid over the harmonic oscillator. This happens because condition (5.2) is not satisfied.

Example $7.2(\mathfrak{s l}(2))$. Finally consider the Lie algebra $\mathfrak{s l}(2)$ with the structure equations

$$
\left[e_{1}, e_{2}\right]=e_{3}, \quad\left[e_{3}, e_{1}\right]=2 e_{1}, \quad\left[e_{2}, e_{3}\right]=2 e_{2}
$$

We can see that the nonzero structure constants are

$$
\stackrel{\circ}{\mu}_{31}^{1}=\stackrel{\circ}{\mu}_{23}^{2}=2 \stackrel{\circ}{\mu}_{12}^{3}=2
$$

System (6.1) reads

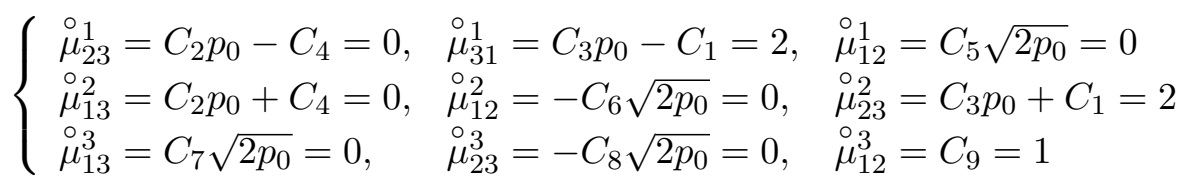

from which it follows that the only nontrivial constants are $C_{3}=\frac{2}{p_{0}}, C_{9}=1$. From (5.3) we get the operadic Lax system

$$
\left\{\begin{array}{l}
\mu_{12}^{1}=\mu_{12}^{2}=\mu_{13}^{3}=\mu_{23}^{3}=\mu_{12}^{3}-1=0 \\
\mu_{23}^{1}=\mu_{13}^{2}=-\frac{2 \omega}{p_{0}} q, \quad \mu_{31}^{1}=\mu_{23}^{2}=\frac{2}{p_{0}} p
\end{array}\right.
$$

\section{Acknowledgement}

The research was in part supported by the Estonian Science Foundation, Grant ETF 6912.

\section{References}

[1] O. Babelon, D. Bernard, and M. Talon. Introduction to Classical Integrable Systems. Cambridge Univ. Press, 2003.

[2] M. Gerstenhaber. The cohomology structure of an associative ring. Ann. of Math. 78 (1963), 267-288.

[3] P. D. Lax. Integrals of nonlinear equations of evolution and solitary waves. Comm. Pure Applied Math. 21 (1968), 467-490.

[4] E. Paal. Invitation to operadic dynamics. J. Gen. Lie Theory Appl. 1 (2007), 57-63.

[5] E. Paal and J. Virkepu. Note on operadic harmonic oscillator. Rep. Math. Phys. 61 (2008), 207-212.

[6] E. Paal and J. Virkepu. 2D binary operadic Lax representation for harmonic oscillator. Preprint arXiv:0803.0592.

Received January 29, 2009

Revised March 03, 2009 